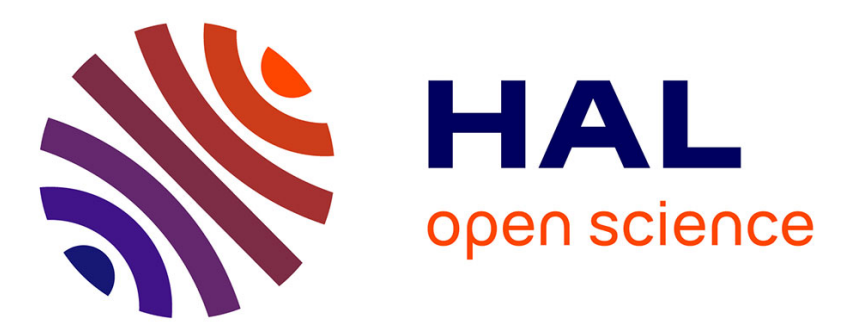

\title{
Les mouvements de capitaux à destination des pays émergents après la crise financière liée aux subprimes
} Jean-Pierre Allegret

\section{To cite this version:}

Jean-Pierre Allegret. Les mouvements de capitaux à destination des pays émergents après la crise financière liée aux subprimes. Développements récents en économie et finances internationales, Mélanges en l'honneur du professeur René Sandretto, Armand Colin, pp.211 - 229, 2012. hal-01410723

\section{HAL Id: hal-01410723 \\ https://hal.parisnanterre.fr/hal-01410723}

Submitted on 15 May 2018

HAL is a multi-disciplinary open access archive for the deposit and dissemination of scientific research documents, whether they are published or not. The documents may come from teaching and research institutions in France or abroad, or from public or private research centers.
L'archive ouverte pluridisciplinaire HAL, est destinée au dépôt et à la diffusion de documents scientifiques de niveau recherche, publiés ou non, émanant des établissements d'enseignement et de recherche français ou étrangers, des laboratoires publics ou privés. 


\title{
Les mouvements de capitaux à destination des pays émergents après la crise financière liée aux subprimes
}

in Développements récents en économie et finances internationales, Mélanges en l'honneur du

professeur René Sandretto, sous la direction de L. Abdelmalki, J.P. Allegret, F. Puech, M.

Sadni Jallab et A. Silem, Armand Colin, Paris, p.211-229, 2012.

\author{
Jean-Pierre Allegret*
}

\section{Introduction}

Les entrées de capitaux dans les pays émergents exercent des pressions sur la balance des paiements appelant des réponses de politique économique ${ }^{1}$. Celles-ci reposent en premier lieu sur l'utilisation des instruments traditionnels à la disposition des autorités. Les autorités peuvent ainsi répondre aux entrées de capitaux par une politique budgétaire restrictive. Cependant, la capacité des autorités à manipuler l'instrument budgétaire peut se heurter à plusieurs obstacles, notamment la position dans le cycle de l'économie concernée, et, d'autre part, la taille de l'Etat. La politique monétaire peut être utilisée dans le sens d'une baisse des taux d'intérêt, mais le risque est alors de laisser se développer des pressions inflationnistes. Enfin, les autorités peuvent choisir de laisser la monnaie domestique s'apprécier. Outre le fait qu'un tel ajustement peut engendrer un cercle vicieux - des anticipations de nouvelles appréciations attirant davantage entrées de capitaux - il peut être contradictoire avec les objectifs de compétitivité.

La réponse aux entrées de capitaux peut reposer en second lieu sur une politique moins traditionnelle fondée sur des restrictions concernant les flux internationaux de capitaux. A la suite de la crise asiatique de 1997, la question du contrôle sur les mouvements internationaux de capitaux a fait l'objet d'intenses débats, tant dans le milieu académique que dans les institutions internationales. Ces dernières, après avoir nettement rejeté les contrôles sur les sorties de capitaux à la malaysienne et avoir exprimé un jugement nuancé sur le contrôle des entrées chilien, le FMI semble admettre l'idée selon laquelle les contrôles peuvent être partie prenante d'une stratégie d'ouverture financière des pays émergents².

La période de la "grande modération » a éclipsé les débats sur l'opportunité de recourir à des contrôles sur les mouvements de capitaux. Ces débats sont revenus sur le devant de la scène avec la crise financière liée aux subprimes et ses développements. D'un côté, la crise a montré une nouvelle fois que les entrées de capitaux sont susceptibles d'engendrer de l'instabilité financière dans les pays récepteurs. De ce point de vue, contrôler les flux de capitaux peut s'interpréter comme un des éléments de la régulation prudentielle. D'un autre côté, on observe depuis le second semestre 2009 un retour des

\footnotetext{
* EconomiX, UMR 7235 CNRS et Université Paris Ouest Nanterre La Défense.

${ }^{1}$ Pour une vue d'ensemble, voir Ghosh et al. (2008).

${ }^{2}$. Il convient ici de souligner la présence des contrôles comme élément de la libéralisation ordonnée des systèmes financiers dans «A guide to progress in strengthening the architecture of the international financial system » disponible sur le site web (http://www.imf.org).
} 
capitaux internationaux dans les pays émergents qui provoquent de fortes tensions sur les changes et les prix. Le contrôle vise alors à essayer de relaxer de telles tensions. Cet article analyse cette résurgence des contrôles en considérant les mouvements à l'œuvre depuis la crise financière.

Dans une première section, les flux de capitaux à destination des pays émergents sont analysés. On montre qu'ils s'inscrivent à la fois dans les principaux cycles traditionnels d'entrées - sorties mais qu'ils revêtent aussi certaines spécificités liées aux conséquences de la crise financière. Dans une seconde section, on souligne que les entrées massives de capitaux conduisent à une accumulation considérable de réserves de change dans les pays émergents. Les distorsions liées à cette accumulation justifient le recours à des mécanismes de contrôle sur les capitaux. La conclusion amorce quelques propositions en termes de réformes du système monétaire et financier international.

1- Anatomie des flux internationaux de capitaux vers les marchés émergents

Le retour des flux de capitaux dans les pays émergents s'inscrit dans un mouvement «classique » de cycles d'entrées - sorties aux effets potentiellement déstabilisants. Cependant, le nouveau cycle qui se dessine après la crise financière de 2007 - 2008 a ceci de spécifique qu'il est en partie le produit des ruptures que cette crise a engendrées à l'échelle mondiale.

1.1 Un mouvement qui s'inscrit dans les différentes vagues d'entrées - sorties de capitaux

Depuis le milieu des années 70, les mouvements internationaux de capitaux à destination des pays émergents ont connu trois cycles principaux (Graphique 1). Ils se caractérisent par des entrées persistantes et un arrêt brutal (Reinhart et Reinhart, 2008 ; IMF, 2010a).

\section{Graphique 1 Flux de capitaux vers les pays émergents, en milliards de dollars}

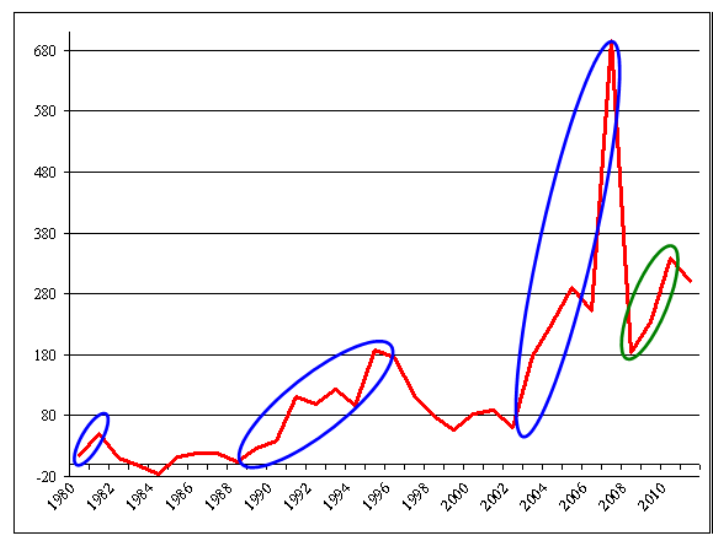

Sources : IMF (2010), World Economic Outlook, base de données, octobre.

Ces cycles d'entrées - sorties de capitaux posent des problèmes macroéconomiques et financiers importants pour les autorités des pays concernés. C'est la motivation principale pouvant justifier le recours au contrôle des capitaux. Il existe une vaste littérature concernant les effets macroéconomiques des flux internationaux de capitaux dans les pays émergents. R. Cardarelli et al. (2009) analysent les périodes d'entrées massives de capitaux pour un échantillon de 52 pays (dont 8 pays développés) sur la 
période $1987-2007^{3}$. Ils montrent, conformément à la littérature antérieure, que les flux de capitaux ont un effet pro-cyclique important. Les entrées nettes s'accompagnent en effet d'une accélération du PIB alors que l'arrêt du cycle d'entrées est suivi par un recul de la croissance. Ce dernier est d'autant plus important que l'épisode d'entrées nettes a duré longtemps.

Cette déstabilisation macroéconomique s'accompagne d'une instabilité financière qui peut renforcer la première. Dans cette perspective, C.M. Reinhart et K.S. Rogoff (2009) ont montré l'existence d'un lien en longue période entre entrées de capitaux et occurrence des crises bancaires (Graphique 2).

\section{Graphique 2 Mobilité internationale des capitaux et incidence des crises bancaires à l'échelle mondiale, 1800 - 2008}

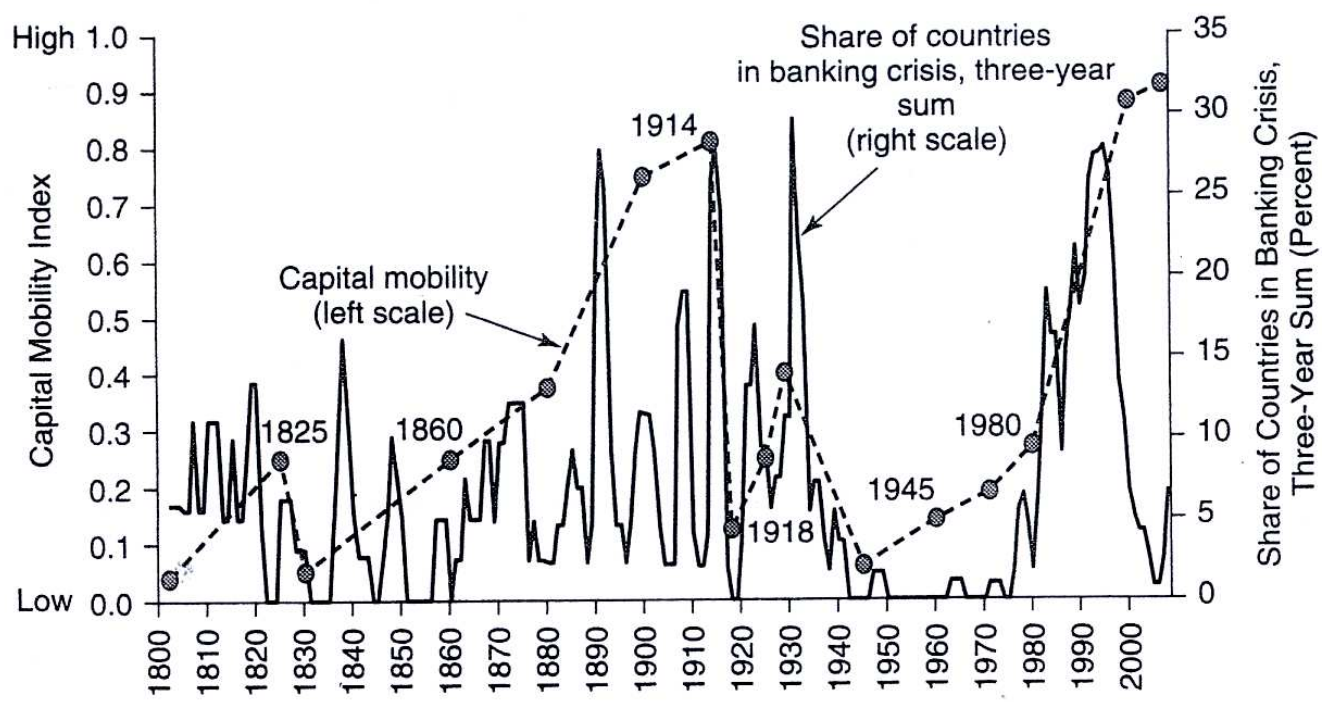

Source : Reinhart et Rogoff (2009), p.156.

Les ressorts de cette instabilité financière sont aujourd'hui bien identifiés. Les entrées de capitaux nourrissent un boom du crédit qui fragilise les bilans bancaires. En outre, les banques domestiques des pays émergents ont tendance à lever des capitaux en devises pour les prêter en monnaie locale conduisant à un déséquilibre de monnaie (currency mismatch) qui les rend très vulnérables à un choc (Kaminsky et Reinhart (1999) ; Rodrik et Velasco (2000)).

\subsection{Un nouveau cycle lié aux ruptures dues à la crise financière}

La crise financière de 2007 - 2008 a induit une accentuation des évolutions observées au sein de l'économie mondiale depuis la fin des années 90. Un trait essentiel de ces évolutions est, d'une part, l'amélioration substantielle des fondamentaux de nombreux pays émergents et, d'autre part, des perspectives de croissance plus importantes de ce groupe de pays par rapport aux pays développés. L'une des ruptures majeures liées à la crise concerne le renversement de la situation des finances publiques entre les deux groupes de pays. Alors que les finances publiques de nombreux pays développés se sont

\footnotetext{
${ }^{3}$ Deux critères sont utilisés pour identifier ces épisodes : (i) un critère spécifique à chaque pays (les entrées nettes en pourcentage du PIB doivent être significativement supérieures au trend) et (ii) un critère régional (les entrées doivent être significativement supérieures à un seuil régional). Un épisode se caractérise par le respect du critère (i) ou du critère (ii) ou des deux à la fois.
} 
très fortement dégradées, celles des pays émergents se sont améliorées. Outre le fait que ces pays n'ont pas été à l'épicentre de la crise financière, un certain nombre d'entre eux a aussi bénéficié du boom des prix des matières premières des années 2003 - 2007. Le corollaire à cette trajectoire opposée des finances publiques est le fait que si, sur la période début 2008 - juin 2010, les pays développés ont connu 25 dégradations de notes de la dette souveraine, les pays émergents ont quant à eux eu 21 améliorations. La crise financière tend aussi à confirmer que, structurellement, les perspectives de croissance des pays émergents sont supérieures à celles des pays développés. Or, dans la mesure où il existe une relation positive entre flux nets de capitaux privés et croissance, il apparaît ici un facteur explicatif structurel aux entrées de capitaux vers les pays émergents.

Dans cette perspective, les meilleures performances relatives des marchés boursiers émergents observées depuis mi-2003 devraient s'accentuer. Ces perspectives de rendements attirent les capitaux internationaux dans un contexte de diversification croissante des portefeuilles des investisseurs. Selon les estimations du FMI (IMF, 2010a), une réallocation de 1 point de pourcentage du portefeuille global d'actions et de titres de dettes des principaux investisseurs internationaux en faveur des pays émergents conduirait à des entrées de capitaux de l'ordre de 485 milliards de dollars, soit un montant supérieur au pic de 2007 (424 milliards).

Un facteur conjoncturel particulièrement puissant pour expliquer le retour des flux internationaux de capitaux vers les pays émergent fait référence aux politiques monétaires menées dans les principaux pays développés depuis le début de la crise financière. Les banques centrales de ces pays se sont lancées dans des politiques de baisses massives des taux d'intérêt qui ont atteint des niveaux historiquement très bas (Graphique 3 panel (a)). Ces politiques ont été rapidement accompagnées par une stratégie quantitative d'aisance monétaire qui s'est traduite par une accélération de la croissance de la masse monétaire à partir du milieu de l'année 2007 (panel (b)). Or, dans la mesure où l'épicentre de la crise s'est situé dans les pays développés, les réactions de politique monétaire dans les pays émergents ont été à la fois de moindre ampleur et de plus courte durée. En conséquence, les taux d'intérêt réels à court terme sont plus élevés dans les marchés émergents relativement aux pays développés (panel (c)). Ainsi, les pays émergents offrent des rendements plus intéressants, ce qui exerce une forte pression en faveur d'entrées massives de capitaux. Autrement dit, une partie significative des entrées de capitaux depuis 2009 s'explique par la politique monétaire menée dans les pays développés.

Graphique 3 Indicateurs de politique monétaire et de liquidités globales 


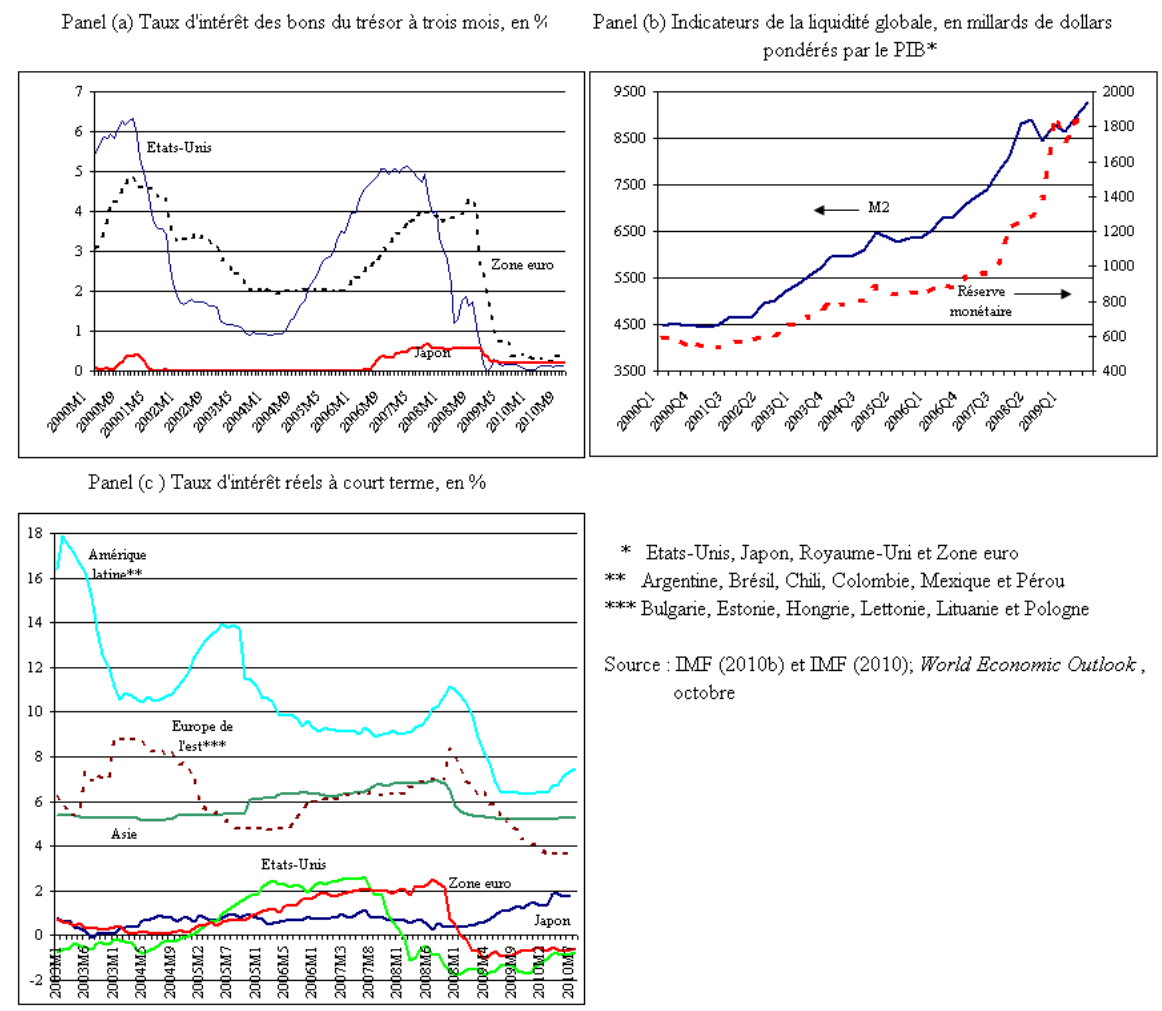

Le FMI (IMF, 2010b) a étudié les conséquences de cette abondance de liquidité globale pour les pays receveurs ${ }^{4}$. Les résultats obtenus suggèrent clairement que les politiques monétaires menées dans les principaux pays développés exercent une influence sur la stabilité financière des pays émergents. Plus précisément, les prix des actions dans les pays émergents tendent à augmenter avec la liquidité globale. Cette dernière conduit aussi à une surévaluation des prix des actions ${ }^{5}$ et à une croissance excessive du crédit domestique ${ }^{6}$. En outre, les flux internationaux de capitaux apparaissent liés à la croissance de la liquidité globale.

Le retour des flux internationaux de capitaux vers les pays émergents repose ainsi sur une imbrication de facteurs structurels et conjoncturels. Quelque soit leur nature, étant donné leur caractère massif, ils posent d'importants problèmes en termes de gestion macroéconomique pour les pays récepteurs. En outre, ces flux internationaux de capitaux restent très dépendants de l'appétit pour le risque des investisseurs internationaux. Autrement dit, même s'ils répondent à des facteurs structurels déjà présents avant la crise, des tensions importantes conduisant à la baisse de l'appétit pour le risque des investisseurs internationaux peuvent conduire à un recul massif de ces flux provoquant alors une autre forme d'instabilité financière.

2- Les réponses de politique économique : entre accumulation des réserves et contrôle des capitaux

Nous concentrons notre attention sur deux types de réponses de la par des autorités : d'une part, les interventions sur les marchés de change et, d'autre part, le recours aux contrôles sur les mouvements de capitaux. Ces deux réponses sont importantes à

\footnotetext{
${ }^{4}$ Les pays étudiés comprennent 37 pays émergents.

${ }^{5}$ Mesurée par la déviation des rendements par rapport à leur moyenne mobile sur un an.

${ }^{6}$ Mesurée par la déviation de la croissance du crédit par rapport à sa moyenne mobile sur un an.
} 
considérer dans la mesure où elles soulèvent toutes les deux des questions liées au fonctionnement du système monétaire et financier international. Elles interrogent aussi les voies de réformes possibles de ce système.

2.1 Entrées de capitaux et accumulation de réserves de change

Comme nous l'avons souligné précédemment, les entrées de capitaux conduisent le plus souvent à des pressions sur les taux de change dans le sens d'une appréciation nominale et / ou réelle de la monnaie domestique. La période postérieure à 2009 n'échappe pas à ce phénomène comme l'illustre le graphique 4 relatif aux taux de change effectifs réels. Le graphique montre que de nombreuses monnaies d'Amérique latine et centrale se sont fortement appréciées en termes réels depuis début 2009 (Brésil, Chili et Mexique, panel (a)). Plus généralement, dans toutes les autres zones émergentes, on relève une tendance à l'appréciation des monnaies à la suite du retour des flux de capitaux (Chine, Indonésie (panel (b)), Russie, Turquie (panel (c)) et Afrique du sud (panel (d))). 
Graphique 4 Taux de change effectifs réels dans quelques pays émergents, $100=$ janvier 2008

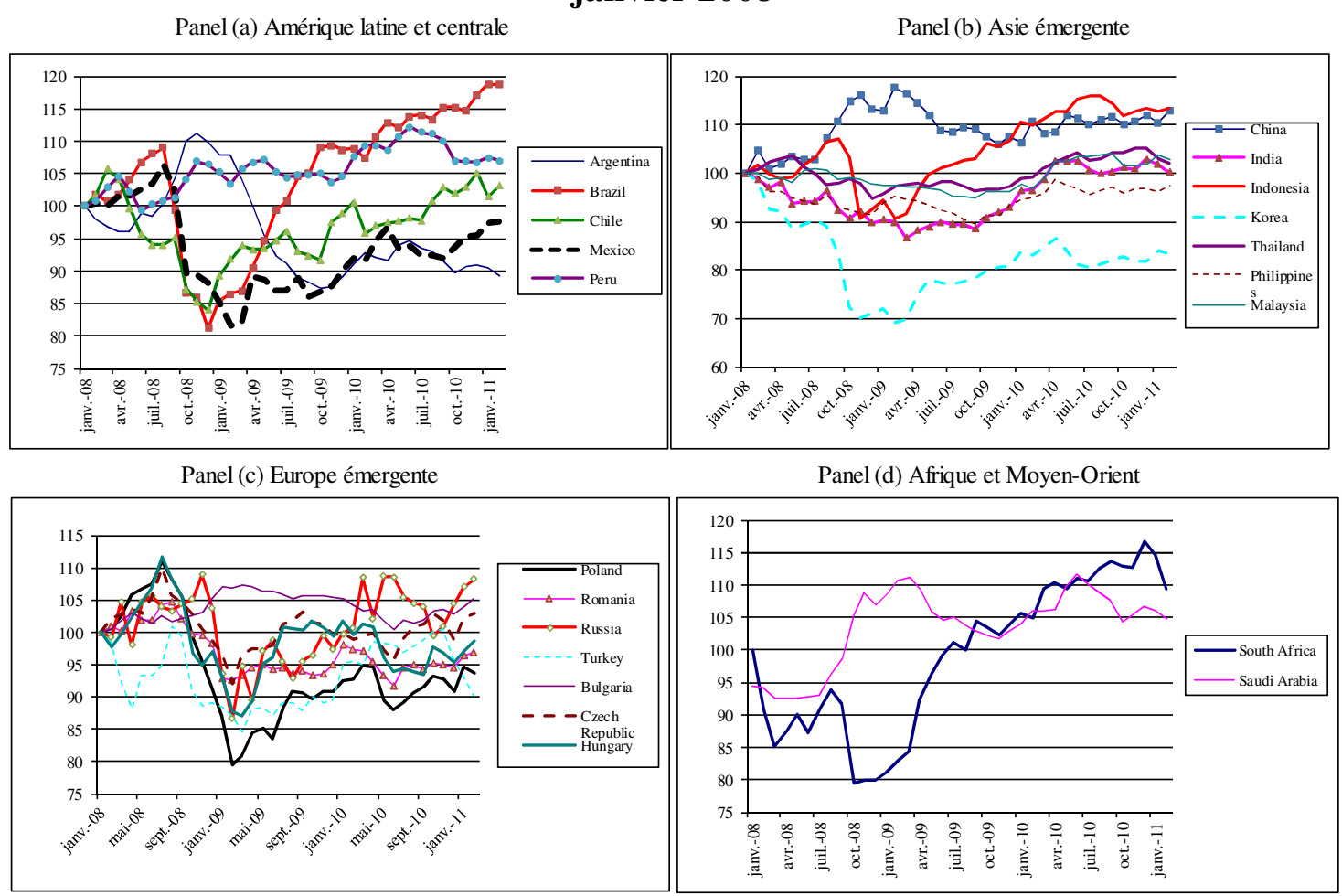

Source : Banque des Règlements Internationaux

Ces pressions sur les changes affectent négativement les pays émergents dont l'activité reste dépendante des exportations et les pays producteurs de matières premières pour lesquelles les prix sont déterminés en dollars.

En conséquence, les autorités de nombreux pays émergents ont répondu à ces pressions par des interventions sur les marchés des changes donnant lieu à une accumulation considérable de réserves de change. La répartition du stock mondial de réserves en devises a été profondément affectée par ces interventions. Le graphique 5 panel (a) montre que l'accumulation de réserves de change a été particulièrement marquée en Asie et dans la région Moyen-Orient et Afrique du nord.

Graphique 5 Réserves en devises dans différentes zones émergentes, 2000 M1 = 100

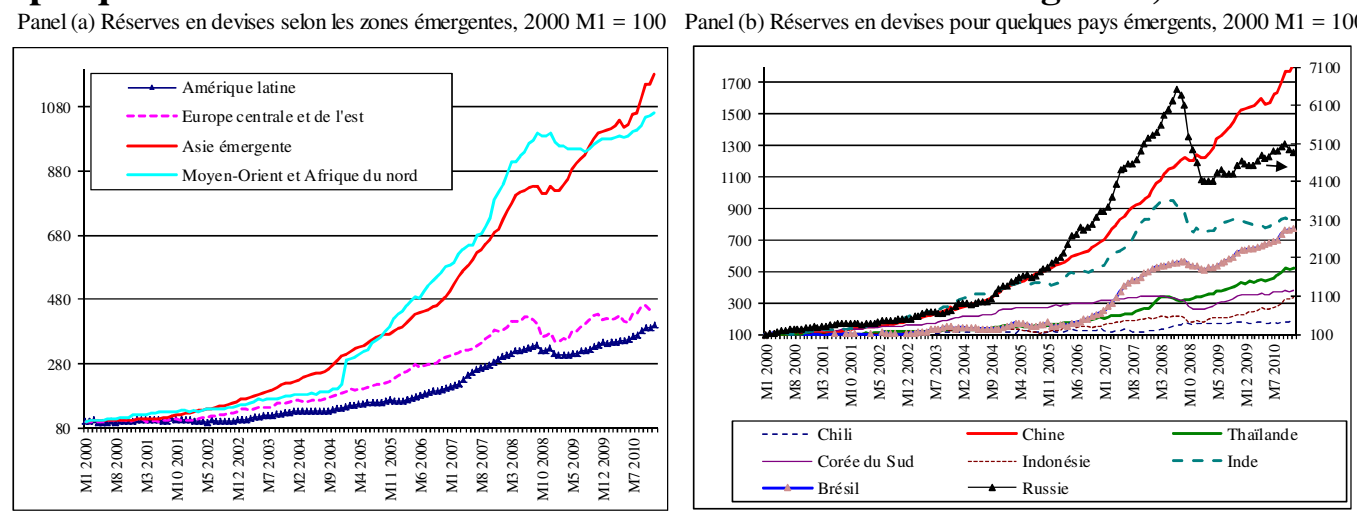

Source : IMF, International financial statistics 
Le panel (b) montre que les BRIC sont les principaux pays ayant accumulé des réserves, notamment après la crise financière de 2007 - 2008. Quelque soit le groupe de pays, on voit que la crise n'a été qu'une pause dans le processus d'accumulation de réserves observé depuis le début des années 2000.

Depuis la crise asiatique de 1997 - 1998, une abondante littérature s'est développée à propos de la politique d'accumulation des réserves de change (Aizenman et Lee, 2007). Un accent particulier a été mis sur le rôle d'auto-assurance joué par les réserves pour les pays émergents. Il s'agit alors de faire face aux crises financières récurrentes liées aux cycles d'entrées - sorties massives de capitaux. Ainsi, en accumulant un montant élevé de réserves, les pays seraient mieux à même de résister à l'instabilité générée par des sorties brutales et massives de capitaux. Si on se situe dans ce cadre, il apparaît assez clairement que les pays émergents détiennent des stocks de réserves en devises bien supérieurs à ce que les ratios traditionnels suggèrent (Smaghi, 2010; Dorrucci et McKay, 2011). Le graphique 6 montre ainsi que le montant des réserves est largement supérieur au ratio habituel des 3 mois d'importations et au respect de la règle Greenspan - Guidotti selon laquelle les réserves de change doivent strictement couvrir le stock de dettes à court terme. En fait, le graphique montre aussi que même avec des ratios plus stricts (6 mois d'importations et un ratio dettes court terme / réserves de $330 \%$ ), le niveau des réserves demeure excessif.

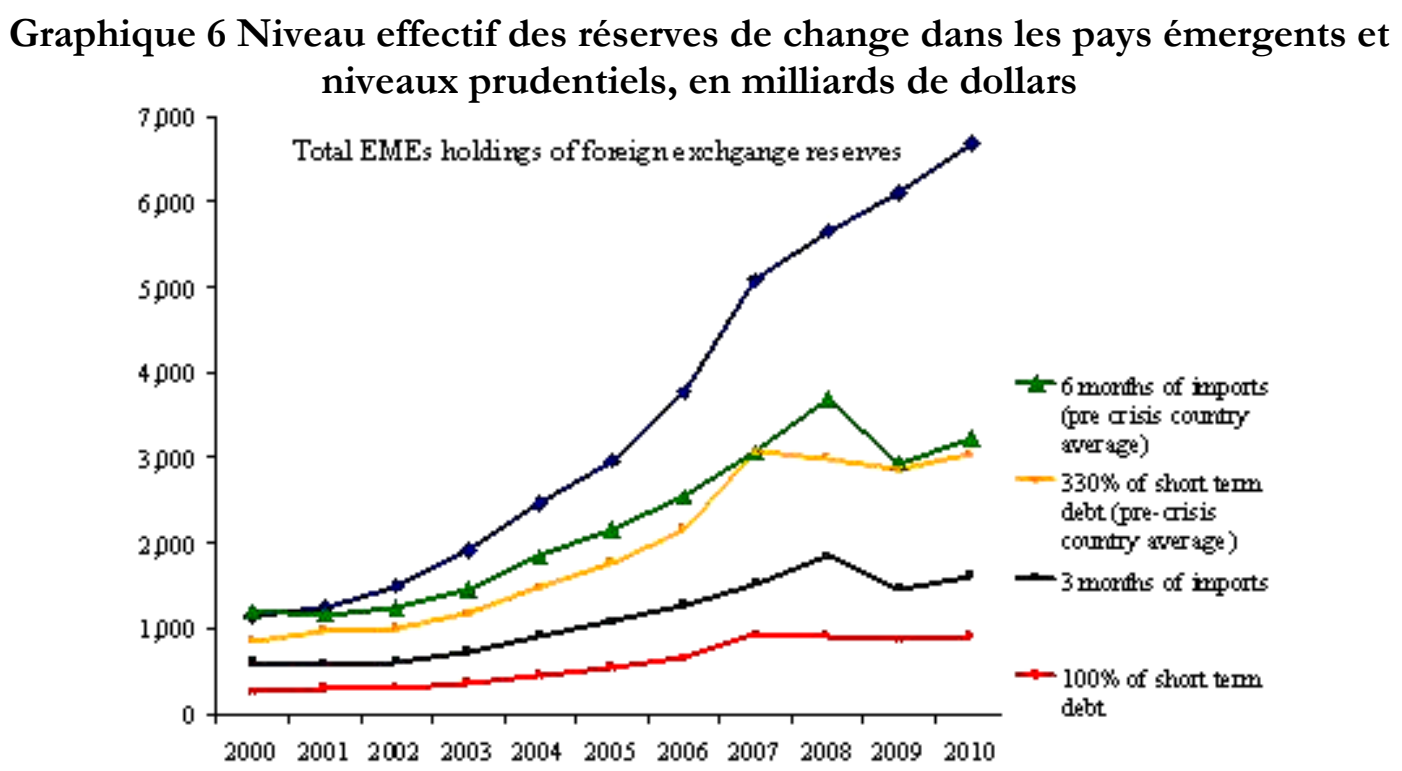

Source : Smaghi (2010)

Si le comportement d'accumulation de réserves soulève de nombreuses questions, c'est notamment en raison de son caractère sous-optimal. On relève en premier lieu une sousoptimalité au niveau domestique. D'une part, le fait que les réserves soient placées dans des actifs sans risque offrant un faible taux de rendement conduit à une sous-utilisation des ressources dans les pays concernés. Plus précisément, pour les économies à excédents courants, l'accumulation de réserves par les banques centrales s'apparente à une épargne nationale intermédiée par le public. Or, cette intermédiation n'est pas optimale dans la mesure où les placements effectués offrent de faibles rendements. D'autre part, par le jeu des contreparties de la masse monétaire, l'accumulation de réserves peut conduire à un accroissement très rapide des agrégats monétaires domestiques. Cet accroissement peut à son tour provoquer des tensions inflationnistes, 
favoriser une expansion excessive du crédit et l'apparition de bulles sur les prix d'actifs. Afin de limiter les effets négatifs de cette accumulation, les autorités recourent souvent à la stérilisation. Les autorités réduisent la composante domestique de la base monétaire (réserves + monnaie en circulation) afin de compenser la hausse de la composante externe (les réserves officielles).

L'inconvénient majeur de cette stratégie réside dans son coût fiscal qui peut devenir exorbitant. En effet, à la suite de ses interventions, la banque centrale détient des actifs de réserve à faible rendement alors qu'elle a dû émettre des titres à haut rendement pour inciter les agents économiques à lui céder la liquidité excédentaire. La charge budgétaire des opérations de stérilisation de la Colombie en 1991 a été estimée à 0,5\% du PIB (Calvo et al., 1993). Cette économie a d'ailleurs été soumise à ce que G.A. Calvo (1991) a qualifié de «péril de la stérilisation » : l'augmentation du stock nominal de dette publique domestique conduit à un accroissement des anticipations d'inflation, ce qui remet en cause la crédibilité du plan de stabilisation. En effet, les agents privés anticipent que les autorités seront incitées à générer à la période suivante de l'inflation surprise pour réduire le poids réel de la dette.

D. Rodrik (2006) a estimé le coût social lié à la détention de réserves de change en considérant la marge entre le rendement sur les actifs liquides de réserves et le coût des emprunts extérieurs pour les agents privés ${ }^{7}$. En excluant de son estimation les réserves détenues pour faire face au financement du compte courant - en prenant comme base de l'estimation une couverture de trois mois d'importations - D. Rodrik obtient un coût social lié à la détention de réserves équivalent à près de $1 \%$ du PIB des pays en développement en 2004. Surtout, ce coût a constamment augmenté depuis le début des années 90 en raison du processus d'accumulation de réserves.

Outre le fait que la stérilisation peut être coûteuse, elle est aussi inefficace pour contenir les effets inflationnistes des entrées de capitaux et l'appréciation de la monnaie en termes réels. En effet, la stérilisation implique que le taux d'intérêt domestique ne change pas. Dès lors, si les entrées de capitaux sont attirées par un taux d'intérêt initialement élevé, cette attirance perdure en l'absence d'une modification de la politique monétaire.

L'accumulation de réserves engendre aussi une sous-optimalité au niveau international. Plus précisément, dans la mesure où la stérilisation n'est pas totale, l'accroissement du stock de réserves détenu par les banques centrales des pays émergents se traduit par une expansion très importante de la liquidité mondiale. Cette abondance de liquidité est un facteur puissant de baisses de rendements à l'échelle internationale, ce qui favorise les comportements spéculateurs de plus en plus risqués. Les investisseurs sont en effet à la recherche de rendements attrayants. R. Beck et M. Fidora (2008) ont recensé 15 études qui estiment l'effet sur les rendements des achats de bons du trésor américains par les

\footnotetext{
${ }^{7}$ Son estimation s'appuie sur le raisonnement suivant. Si une entreprise décide d'emprunter x dollars sous la forme d'un crédit extérieur à court terme, la banque centrale devra détenir x dollars de réserves en plus afin de respecter la règle de Greenspan - Guidotti. La banque centrale achète sur le marché domestique pour x dollars de réserves et les place aux Etats-Unis. Si elle stérilise son accumulation de réserves, elle vend au secteur privé domestique pour x dollars de titres publics domestiques. En termes de bilan agrégé de l'économie, l'effet net est tel que l'emprunt étranger à court terme (financé au coût d'emprunt du secteur privé) a pour contrepartie un investissement dans un actif étranger de court terme. La différence entre les taux d'intérêt représente le coût social de la détention de devises.
} 
banques centrales étrangères. La majorité des études situe cet effet dans une marge allant de 20 à 100 points de base ${ }^{8}$.

\subsection{La résurgence des contrôles sur les flux internationaux de capitaux}

Le retour des entrées massives de capitaux dans les pays émergents s'est accompagné d'une résurgence des mesures destinées à contrôler les flux internationaux de capitaux. L'annexe 1 présente un certain nombre de mesures prises depuis octobre 2009 dans les principaux pays émergents. Si ces derniers ont dans l'ensemble adopté une grande variété de mesures, on peut néanmoins les regrouper en quatre familles principales : (i) utilisation de taxes (Brésil, Corée du sud, Pérou, Russie et Thaïlande) ; (ii) instauration de périodes minimales d'investissement (Indonésie); (iii) les mesures quantitatives soit dans un sens restrictif (Chine, Corée du sud, Indonésie, Pérou et Turquie), soit dans un sens moins restrictif afin de faciliter les sorties de capitaux (Afrique du sud, Philippines et Thaïlande) ; (iv) les réserves non rémunérées (Brésil, Pérou et Turquie).

L'efficacité des mesures adoptées dans les économies émergentes peut être abordée à partir des objectifs initialement visés : (i) action sur le volume et la composition des entrées de capitaux; (ii) maîtrise des taux d'intérêt domestiques; (iiii) contrôle de l'appréciation du taux de change réel.

Sur tous ces objectifs, la littérature antérieure aux années 2000 (Allegret, 2000) laisse une impression mitigée. Ainsi, C.M. Reinhart et R.T. Smith (1998) - en étudiant cinq marchés émergents (Chili, Colombie, Malaisie, Brésil et République tchèque) parviennent à la conclusion selon laquelle le Chili et la Malaisie (et dans une mesure moindre la République Tchèque) ont vu le volume des entrées de capitaux se réduire immédiatement après l'introduction d'un contrôle. Cependant, il ne s'agit pas à proprement parler d'une rupture dans la tendance des flux de capitaux. Cela signifie que l'introduction du contrôle ne parvient à réduire que temporairement le volume des entrées de capitaux. On observe par contre les effets désirés d'un contrôle sur la composition des entrées de capitaux : leur maturité s'accroît significativement au Chili, en Colombie et en Malaisie. Cependant, si on considère - en suivant en ce sens $\mathrm{S}$. Edwards (1999) - les données relatives à la période d'avant la crise asiatique, alors il apparaît que la situation chilienne n'est pas très différente de celle de pays sans contrôle tels l'Argentine et le Mexique. En ce qui concerne la capacité d'un contrôle à maintenir un écart d'intérêt significatif entre les taux d'intérêt, S. Edwards (1998) a étudié le comportement de cet écart au Chili en distinguant la période antérieure au contrôle (1986 - 1991) et la période postérieure $(1991$ - 1996). En utilisant un modèle VAR, il parvient à la conclusion selon laquelle le contrôle permet un gain d'autonomie de la politique monétaire, mais celui-ci ne perdure pas au cours du temps. Autrement dit, le gain en indépendance monétaire, s'il est présent, n'est que de courte durée. L’impact du contrôle sur le comportement du taux de change réel - autre objectif central des économies émergentes - conduit à des conclusions encore plus négatives quant à l'efficacité des mesures de restriction sur la mobilité des capitaux (Edwards, 1998 ; Cowan et De Gregorio, 1998 ; Reinhart et Smith, 1998).

\footnotetext{
8 Ces estimations ne tiennent pas compte que des seules banques centrales des pays émergents. Cependant, il convient de noter qu'en décembre 2005, la part des pays émergents et pétroliers dans la détention étrangère de bons du Trésor américain s'élevait à 35,5\%. En janvier 2011, cette part se situait à $53,5 \%$. Calculs effectués sur la base des données fournies par le Département du Trésor (http://www.treasury.gov/resource-center/data-chart-center/tic/Pages/ticsec2.aspx\#ussecs ).
} 
Les travaux postérieurs à l'année 2000 confirment en partie la littérature précédente tout en montrant une certaine efficacité des contrôles. M. Binici et al. (2009) utilisent une nouvelle base de données permettant de distinguer les effets des contrôles à l'entrée et à la sortie de manière désagrégée. Etudiant 74 pays sur la période 1995 - 2005 à l'aide de l'économétrie des données de panel ${ }^{9}$, ils montrent que si les contrôles exercent une influence sur les flux globaux de capitaux, cela est essentiellement dû aux effets des contrôles sur les investissements en actions (y compris les investissements directs étrangers). Surtout, les contrôles ont peu d'effets sur les entrées, alors que, contrairement à ce que suggèrent d'autres études, ils permettent de limiter les sorties de capitaux. Dans un travail recensant une trentaine de travaux publiés entre 1995 et 2004, N.E. Magud et al. (2011) parviennent à la conclusion selon laquelle, si les contrôles n'ont pas d'effets sur le volume des flux de capitaux, ils parviennent à allonger la maturité des entrées, à donner des gains d'indépendance monétaire et à réduire les pressions sur les taux de change réel. Cependant, l'efficacité des contrôles repose sur les conditions initiales, notamment les politiques économiques sous-jacentes menées dans les pays concernés (voir aussi Allegret, 2000). Les auteurs soulignent aussi que l'efficacité des contrôles sur les sorties de capitaux est limitée, à l'exception de l'expérience malaise de 1998.

Etudiant sur données journalières entre 1991 et 1998 l'expérience chilienne, S. Edwards et R. Rigobon (2009) utilisent une méthodologie permettant d'analyser l'efficacité du contrôle alors que dans le même temps les autorités interviennent sur le marché des changes. Leurs résultats sont particulièrement intéressants dans la mesure où ils obtiennent que, d'une part, un durcissement du contrôle conduit à la dépréciation de la monnaie domestique et que, d'autre part, il réduit la vulnérabilité du taux de change aux chocs externes. J.D. Ostry et al. (2010) trouvent une relation négative entre la présence de contrôles sur les capitaux avant la crise financière liée aux subprimes et la chute de la production durant la crise. Ils soulignent notamment que les contrôles agissant sur les déterminants de la vulnérabilité financière - limitation des entrées de capitaux conduisant à un boom du crédit et un accroissement des prêts bancaires en devises sont particulièrement efficaces. Ces résultats sont en ligne avec ceux de P. Gupta et al. (2007). Ces derniers analysent le comportement de la production durant 195 épisodes de crises de change sur la période 1970-2000 et pour un échantillon de 91 pays en développement. Ils montrent entre autres que la contraction de la production durant la crise est d'autant plus importante que le pays a reçu des flux élevés de capitaux avant l'occurrence de cette crise. Introduisant un indice de restriction sur les transactions au titre du compte de capital comme terme d'interaction avec les flux d'entrées de capitaux, ils obtiennent que la présence de restrictions avant la crise réduit significativement le recul de la production après la crise ${ }^{10}$.

Etudiant les expériences de contrôle de cinq pays émergents entre 2000 et $2008^{11}$ à l'aide d'un modèle VAR, le FMI (IMF, 2010b) montre que les contrôles sont associés à une baisse des entrées de capitaux et à un allongement de leur maturité, qu'ils ne

\footnotetext{
${ }^{9}$ Il convient de préciser que les auteurs utilisent des variables de contrôle explicatives des flux de capitaux: PIB réel par tête, qualité institutionnelle, taux d'ouverture commerciale, indicateurs de développement financier et présence de ressources naturelles.

${ }^{10}$ A contrario, Cardarelli et al. (2009) ne trouvent aucun lien entre la présence ou non d'un contrôle sur les entrées de capitaux et la chute de la production après une crise financière.

${ }^{11}$ Brésil (2008), Colombie (2007-2008), Croatie (2006-2007) et Thaïlande (2006-2008) pour les entrées et Corée du Sud (2005-2008) pour les sorties.
} 
permettent pas de limiter l'appréciation de la monnaie en termes réels et qu'ils procurent des gains temporaires du point de vue de l'autonomie monétaire.

Conclusion

Le retour des flux de capitaux dans les pays émergents confrontent ces derniers à d'importants défis : d'une part, défis en termes de gestion macroéconomique (contenir les pressions inflationnistes et sur le change) ; d'autre part, défis du point de vue de la stabilité financière (éviter l'occurrence d'un boom du crédit ou du bulles de prix d'actifs). L'après-crise financière - en engendrant d'importants écarts de croissance et de taux d'intérêt entre les zones - tend à aggraver l'ampleur de ces défis. Les réponses de politique économique dans de nombreux pays émergents ont été fondées principalement sur l'accumulation de réserves de change et / ou le recours à des mesures de contrôles de capitaux.

Les limites de ces réponses ont relancé les débats - souvent anciens - sur la réforme du système monétaire international. Un certain nombre de propositions se sont focalisés sur les dispositifs à mettre en œuvre afin de limiter l'accumulation de réserves à des fins de précaution. Ces propositions convergent pour promouvoir la création d'un filet mondial de sécurité financière (global financial safety net) (Mateos y Lago et al., 2009 ; IMF, 2010c ; Dorrucci et McKay, 2011). De telles propositions se heurtent aux limites du système monétaire international actuel déjà amplement débattues dans les années 90 : absence d'automaticité dans l'accès aux moyens financiers en raison de la conditionnalité et / ou des critères de pré-qualification et absence de prêteur international en dernier ressort interdisant toute création illimitée de liquidité à l'échelle mondiale. L'action sur l'accumulation de réserves pour des motifs autres que celui de précaution s'inscrit dans une réforme plus vaste du système monétaire international. Elle passe à la fois par une politique de coordination internationale visant à réduire l'ampleur des déséquilibres mondiaux et par la promotion d'un système de réserves plus diversifié, voir la création d'une nouvelle unité de réserve.

La multiplication des mesures unilatérales de contrôle sur les capitaux a aussi remis en lumière l'absence de cadre multilatéral intégrant la question de la mobilité internationale des capitaux (IMF, 2010d). A cette fin, M. Camdessus et al. (2011) préconisent d'amender l'article VI des Accords de Bretton Woods afin de doter le Fonds monétaire international des responsabilités liées à la surveillance des mouvements de capitaux et à celle du recours aux restrictions par les pays membres. L'initiative n'est pas nouvelle puisqu'elle avait déjà été évoquée lors de la réunion annuelle du FMI à Hong Kong en septembre 1997 (Fischer et al., 1998). Un tel amendement en la matière pourrait cependant avoir deux avantages importants (Allegret et Sandretto, 2000 ; Allegret et Dulbecco, 2002). D'une part, l'intégration financière internationale induisant des forces centripètes, des pays peuvent être incités à s'ouvrir financièrement alors qu'ils ne sont pas prêts. Compte tenu des externalités négatives liées à l'intégration financière, une telle stratégie peut se révéler déstabilisante pour le système financier international. D'autre part, le recours aux contrôles peut être interprété par les investisseurs comme une remise en cause de la stratégie de libéralisation financière. Un tel signal négatif est alors susceptible de provoquer une fuite massive de capitaux et/ou de conduire à une augmentation du coût des emprunts sur les marchés internationaux de capitaux pour le pays considéré. Les remarques précédentes conduisent à privilégier une approche dans laquelle est il explicitement reconnu que le recours aux contrôles de capitaux est une stratégie permettant 
d'améliorer la stabilité financière. Dans cette perspective, le Fonds pourrait être amené à préciser les conditions de leur utilisation.

Ainsi, les développements de l'après-crise financière internationale ont conduit, non pas à un renouvellement des analyses en matière de réformes, mais plutôt à une réactivation de problèmes anciens que les précédentes crises financières avaient fait émerger. La question reste ouverte de leur réelle mise en œuvre.

Références

Aizenman J. et Lee J. (2007), «International reserves : precautionary versus mercantilist views, theory and evidence », Open Economies Review, 18 : 191-214.

Allegret J.P. (2000), «Quel rôle pour les contrôles des mouvements internationaux de capitaux ? », Economie internationale, 81 : 77-108.

Allegret J.P. et Sandretto R. (2000), «La nouvelle architecture du système monétaire international. Revenir à l'esprit des lois de Bretton Woods », L'actualité économique, Revue d'analyse économique, 76 (3) : 437-456.

Allegret J.P. et Dulbecco P. (2000), «Global governance versus domestic governance : what roles of international institutions? », European Journal of Development Research, 14 (1) : 263-272.

Beck R. et Fidora M. (2008), The impact of sovereign wealth funds on global financial markets, Intereconomics, 43 (6) : 349-358.

Binici M., Hutchison M. et Schindler M. (2009), "Controlling capital ? Legal restrictions and the asset composition of international financial flows », IMF Working Paper, WP/09/208, septembre.

Calvo G.A. (1991), « The peril of sterilization », IMF Staff Papers, 38 (4) : 921-926.

Calvo G.A., Leiderman L. et Reinhart C.M. (1993), « Capital inflows and real exchange rate appreciation in Latin America, the role of external factors », IMF Staff Papers, 40 (1) : 108-151.

Camdessus M., Lamfalussy A. et Padoa-Schioppa T. (2011), Reform of the international monetary system : a cooperative approach for the twenty first century, février.

Cardarelli R., Elekdag S. et Kose M.A. (2009), «Capital inflows : macroeconomic implications and policy responses », IMF Working Paper, WP/09/40, mars.

Cowan K. et De Gregorio (1998), "Exchange rate policies and capital account management: Chile in the 1990s », in R. Glick (ed.), Managing capital flows and exchange rates, perspectives from the Pacific Basin, Cambridge University Press, 465488.

Dorrucci E. et McKay J. (2011), «The international monetary system after the financial crisis », ECB, Occasional Paper Series, 123, février.

Edwards S. (1998), «Capital flows, real exchange rates, and capital controls : some Latin American experiences », NBER Working Paper Series, 6800, novembre.

Edwards S. (1999), «How effective are capital controls?», NBER Working Paper Series, 7413, novembre.

Edwards S. et Rigobon R. (2009), « Capital controls on inflows, exchange rate volatility and external vulnerability », Journal of International Economics, 78 : 256-267. 
Ghosh A., Goretti M., Joshi B., Ramakrishnan U., Thomas A. et Zalduendo J. (2008), «Capital inflows and balance of payments pressures - Tailoring policy responses in emerging market economies », IMF Policy Discussion Paper, PDP/08/2, juin.

Gupta P., Mishra D. et Sahay R. (2007), « Behavior of output during currency crises », Journal of International Economics, 72 (2) : 428-450.

International Monetary Fund (2010a), Global financial stability report, octobre.

International Monetary Fund (2010b), «Global liquidity expansion: effects in "receiving" economies and policy responses options », Global financial stability report, avril, chapitre 4 .

International Monetary Fund (2010c), Reserve accumulation and international monetary stability, Strategy, Policy and Review Department, avril.

International Monetary Fund (2010d), The Fund's role regarding cross-border capital flows, Strategy, Policy, and Review Department and the Legal Department, novembre.

Kaminsky G.L. et Reinhart C.M. (1999), «The twin crises : the causes of banking and balance-of-payments problems », The American Economic Review, 89 (4) : 473-500.

Magud N.E., Reinhart C.M. et Rogoff K.S. (2011), «Capital controls : myth and reality - A portfolio balance approach », NBER Working Paper, 16805, février.

Mateos y Lago I., Duttagupta R. et Goyal R. (2009), « The debate on the international monetary system », IMF Staff Position Note, SPN/09/26, novembre.

Ostry J.D., Ghosh A.R., Habermeier K., Chamon M., Qureshi M.S. et Reinhardt D.B.S. (2010), «Capital inflows : the role of controls », IMF Staff Position Note, SPN/10/04, février.

Reinhart C.M. et Reinhart V.R. (2008), «Capital flows bonanzas : an encompassing view of the past and present », NBER Working Paper Series, 14321, septembre.

Reinhart C.M. et Rogoff K.S. (2009), This time is different : eight centuries of financial folly, Princeton University Press.

Reinhart C.M. et Smith R.T. (1998), « Too much of a good thing : the macroeconomic effects of taxing capital inflows », in R. Glick (ed.), Managing capital flows and exchange rates, perspectives from the Pacific Basin, Cambridge University Press, 436464.

Rodrik D. (2006), "The social cost of foreign exchange reserves », International Economic Journal, 20 (3) : 253-266.

Rodrik D. et Velasco A. (2000), «Short-term capital flows », in Annual World Bank Conference on Development Economics, Washington D.C.

Smaghi L.B. (2010), «Reserve accumulation : the other side of the coin », $5^{\text {ème }}$ Highlevel EMEAP-Eurosystem Seminar, Sydney, février. 
Annexe 1 Principales mesures de contrôles sur les mouvements internationaux dans les pays émergents depuis octobre 2009

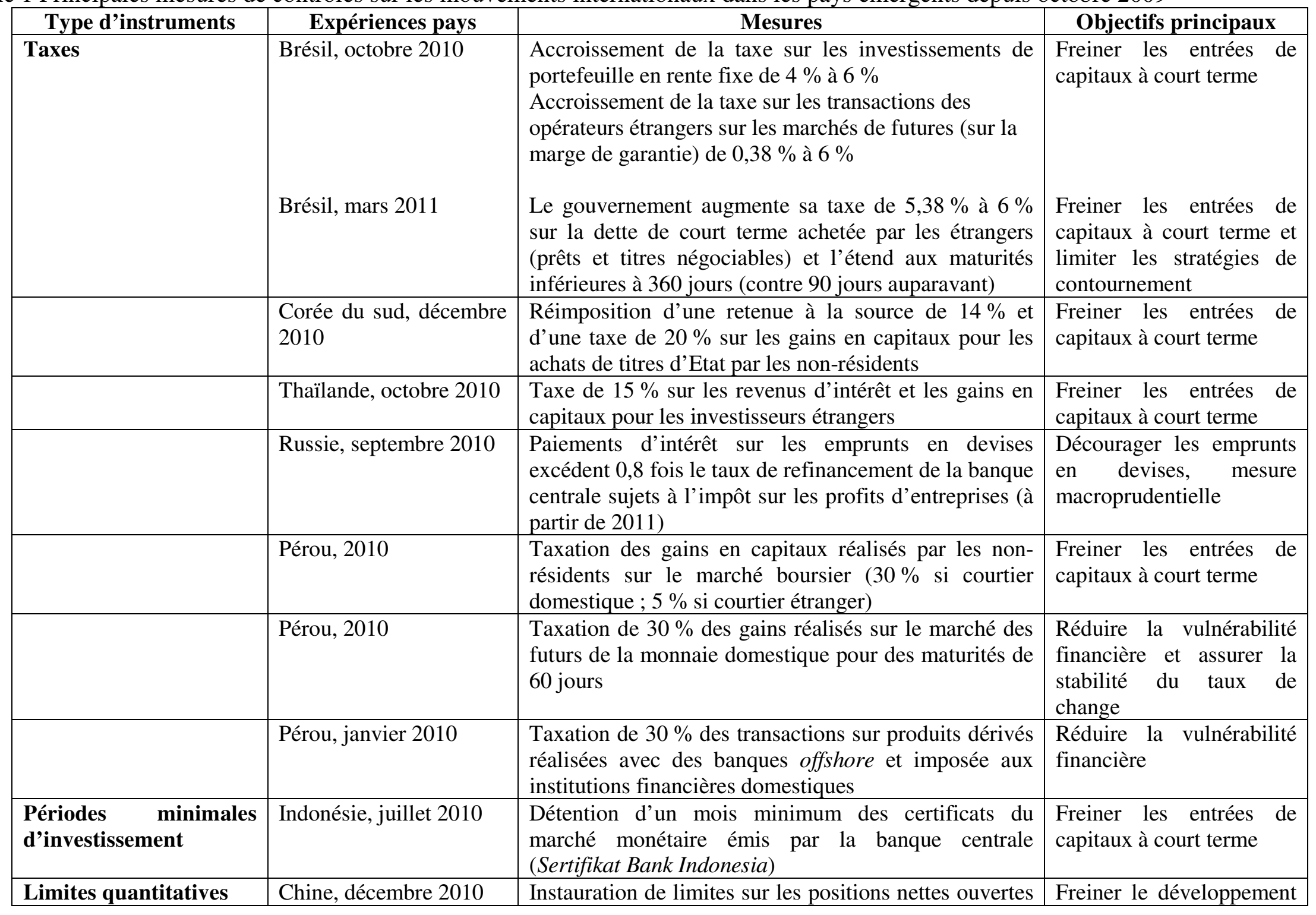




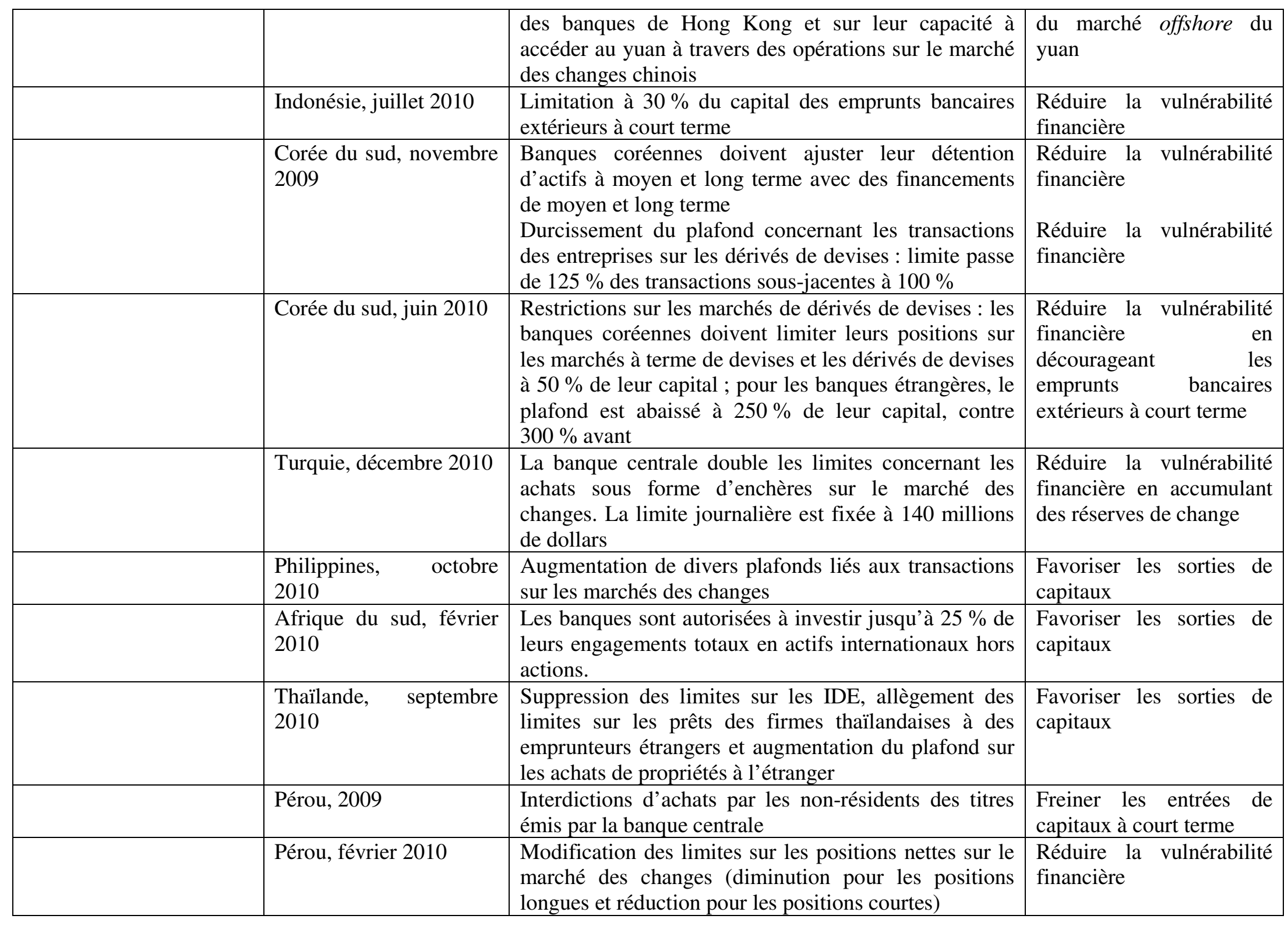




\begin{tabular}{|c|c|c|c|}
\hline & Pérou, septembre 2010 & $\begin{array}{l}\text { Augmentation du plafond des investissements } \\
\text { internationaux des fonds de pension privés }\end{array}$ & $\begin{array}{lll}\begin{array}{l}\text { Favoriser les sorties de } \\
\text { capitaux }\end{array} & & \\
\end{array}$ \\
\hline & Pérou, juin 2010 & $\begin{array}{l}\text { Introduction de limites sur les transactions des fonds } \\
\text { de pension privés sur le marché des changes }\end{array}$ & $\begin{array}{l}\text { Réduire la vulnérabilité } \\
\text { financière et assurer la } \\
\text { stabilité du taux de } \\
\text { change }\end{array}$ \\
\hline \multirow[t]{3}{*}{$\begin{array}{l}\text { Réserves } \\
\text { rémunérées }\end{array}$} & Brésil, janvier 2011 & $\begin{array}{l}\text { Réserve de } 60 \% \text { sur les positions courtes en dollars } \\
\text { des banques sur le marché des changes au comptant }\end{array}$ & $\begin{array}{l}\text { Freiner les entrées de } \\
\text { capitaux à court terme }\end{array}$ \\
\hline & Turquie, décembre 2010 & $\begin{array}{l}\text { Accroissement des réserves et extension aux } \\
\text { transactions liées aux prises en pension } \\
\text { Différenciation sur la maturité : augmentation des } \\
\text { exigences pour les maturités inférieures à un an ; } \\
\text { réduction pour les maturités supérieures à un an }\end{array}$ & $\begin{array}{l}\text { Freiner les entrées de } \\
\text { capitaux à court terme }\end{array}$ \\
\hline & Pérou, 2009 & $\begin{array}{l}\text { Accroissement des exigences de réserves sur tous les } \\
\text { dépôts. Le montant des réserves s'élève à } 120 \% \text { pour } \\
\text { les dépôts en monnaie domestique détenus par les non- } \\
\text { résidents. } \\
\text { Les exigences de réserves montent à } 75 \% \text { pour les } \\
\text { autres engagements étrangers de maturité inférieure à } \\
\text { deux ans }\end{array}$ & $\begin{array}{l}\text { Freiner les entrées de } \\
\text { capitaux à court terme }\end{array}$ \\
\hline
\end{tabular}

Sources : Magud et al. (2011), IIF (2011), Capital Markets Monitor, special feature II : Update on recent capital control measures, janvier, IIF (2011), Singh K. (2010), Emerging markets consider capital controls to regulate speculative capital flows, Vox, juillet, HSBC (2010), Manning the barricades, the return of capital controls and the implications for global markets, HSBC Global Research, novembre, South African Reserve Bank, Central Bank of the Republic of Turkey. 